\title{
Optimal Approximate Inverse of Linear Periodic Filters
}

\author{
Jwo-Yuh Wu and Ching-An Lin
}

\begin{abstract}
We propose a method for constructing optimal causal approximate inverse for discrete-time single-input single-output (SISO) causal periodic filters in the presence of measurement noise. The analysis is based on block signals and multi-input multi-output (MIMO) time-invariant models for periodic filters. The objective function to be minimized is the asymptotic block mean square error. The optimization problem is formulated in terms of transfer matrices as an optimal model-matching problem with nonsquare model and plant. Based on an inner-outer factorization on the transpose of the plant rational matrix, it is shown that the problem can be further reduced to one with a lower dimensional square model and plant, which is then solved in the time-domain, and a closed-form solution is obtained. A lower bound on the objective function is given. It is shown that the lower bound can be asymptotically achieved as the order of the optimal transfer matrix increases. The proposed method is extended to MIMO periodic systems. Numerical examples are used to illustrate the performance of the proposed approximate inverse.
\end{abstract}

Index Terms-Approximate inverse, block signal processing, deconvolution, inner-outer factorization, inverse, optimal model-matching, periodic filters.

\section{INTRODUCTION}

$\mathbf{P}$ ERIODIC filters and systems have been found useful in the areas of signal processing and communications, e.g., in subband coding [20], in transmultiplexer modeling and design [16], in speech scrambling [9], in co-channel interference cancellation [4], and in blind equalization [15], [17]. The inverse, or approximate inverse, of periodic filters is used for recovering the scrambled speech signals [9] and for equalization of periodically time-varying channels [17], [24]. Inverting periodic filters is discussed in [8], [10], and [21] for noiseless case and, recently, in [23] and [24] when measurement noise is present.

It is well known that associated with each $N$-periodic filter, there is an $N$-input $N$-output time-invariant system that exhibits an input-output $(\mathrm{I} / \mathrm{O})$ relation that is identical to that of the filter [7], [13], [20]. This MIMO time-invariant model corresponds to the block filter implementation [20, p. 428]. For a general study of periodic systems, in particular, in the inverse filtering problem, this model is often adopted since the time-invariant

Manuscript received June 13, 2002; revised September 16, 2003. This work was supported by the National Science Council under Grant NSC-89-2213E009-218. The associate editor coordinating the review of this manuscript and approving it for publication was Dr. Karim Abed-Meraim.

J. Y. Wu is with the Department of Communication Engineering, National Chiao Tung University, Hsinchu, Taiwan, R.O.C. (e-mail: jywu@cc.nctu.edu.tw)

C.-A. Lin is with the Department of Electrical and Control Engineering, National Chiao Tung University, Hsinchu, Taiwan, R.O.C (e-mail: calin@cc.nctu.edu.tw).

Digital Object Identifier 10.1109/TSP.2004.831920 nature would allow considerable simplification in problem formulation as well as in analysis and design. Indeed, based on such a model, there is a very simple procedure for determining the invertibility condition in the absence of noise [8], [10]; also, the well-established design techniques for time-invariant systems can be used for inverse filter design [10], [23]. It is known that such a filter model must satisfy an additional structure constraint owing to causality [7, p. 1090]. As a result, the inverse filter design problem thus formulated will be closely related to constructing an appropriate "inverse" time-invariant system subject to this constraint to guarantee the existence of a corresponding single-input single-output (SISO) causal periodic realization (as is required in real-time operations). In the noiseless case, Lin and King [10] proposed a method for finding such an "inverse" transfer matrix; however, the stability issue of the solution toward a causal periodic implementation has yet to be addressed. For the general case when noise is present, there has yet to be a related study of approximate inverse design based on such MIMO time-invariant formulation (see the introductory comments in [24, p. 1685-1686]). Zhang et al. in [23] and [24], on the other hand, propose an alternative "direct" approach: They formulate the problem directly in terms of the state equation for periodic filters; the optimal inverse filter coefficients are computed, in an iterative manner, based on the linear-matrix-inequality (LMI) method.

This paper is a generalization of the previous work in [10]. Based on block signals and MIMO time-invariant models for periodic filters, we study the problem of constructing an approximate inverse for a given periodic filter [either infinite impulse response (IIR) or finite impulse response (FIR)] when there is noise. For a given reconstruction delay, we show how to construct an approximate inverse, which is a causal and stable periodic filter with the same period, such that the average energy of the block reconstruction error is kept small. There is a natural formulation of the optimization problem in terms of transfer matrices of periodic filters as an optimal model-matching problem [2], [5]. Owing to the noise effect, it is seen that the resultant model and plant are nonsquare rational matrices. Based on an inner-outer factorization on the transpose of the plant rational matrix, it is shown that the problem is further reduced to one with lower dimensional square model and plant. The reduction in dimensions of model and plant using inner-outer factorization leads to a simple procedure for obtaining an optimal transfer matrix subject to the causality constraint; it also enables us to obtain a lower bound on the objective function. Compared with the LMI-based method [23], [24], our solution is in closed form. With the optimal solution, it can be shown that the objective function can be made arbitrarily close to the obtained 
lower bound if a sufficiently large reconstruction delay is allowed. Our formulation, moreover, allows a direct extension of the proposed method for constructing an MIMO approximate inverse of a given MIMO periodic system.

The organization of this paper is as follows. Section II is the problem statement and some preliminary results. Section III proposes the optimality criterion. Section IV shows that the problem is equivalent to solving an optimal model-matching problem and gives a lower bound on the objective function. The construction of the solution is given in Section V. Section VI extends the proposed method to MIMO periodic systems. Section VII is the simulation results. Finally, Section VIII is the conclusion.

List of Notations: Let $\Re^{l \times m}$ be the set of all $l \times m$ real matrices. The notations $0_{l \times m}$ and $I_{l}$, respectively, stand for the $l \times m$ zero matrix and the $l \times l$ identity matrix. We say a real rational matrix $\mathbf{X}(z)$ is proper if $\mathbf{X}(\infty)$ is a constant matrix with finite entries. Let $\Re_{p}^{l \times m}(z)$ be the set of all $l \times m$ proper real rational matrices in $z$ with all its poles in the open unit disk $\{z:|z|<1\}$. The notation $\partial D$ denotes the unit circle $\{z:|z|=1\}$. Let $\Re^{l \times m}(z)$ be the set of all $l \times m$ real rational matrices in $z$ with no poles on $\partial D$. The convolution of two sequences $\mathbf{X}, \mathbf{Y}$ is defined as $\mathbf{X} * \mathbf{Y}=\sum_{m=-\infty}^{\infty} \mathbf{X}_{m} \mathbf{z}^{-m} \mathbf{Y}$, where $\mathbf{z}^{-m}$ is the $m$-step delay such that $\left(\mathbf{z}^{-m} \mathbf{Y}\right)_{k}=\mathbf{Y}_{k-m}$. The notation $E a$ denotes the expected value of the random variable $a$.

\section{PRoblem Statement AND PRELIMINARY}

\section{A. Problem Statement}

Consider the discrete-time linear causal $N$-periodic filter described by the following state equation ${ }^{1}$

$$
\begin{aligned}
x_{k+1} & =A_{k} x_{k}+b_{k} s_{k} \\
u_{k} & =c_{k} x_{k}+d_{k} s_{k}, \quad k \geq 0
\end{aligned}
$$

where $s_{k} \in \Re, u_{k} \in \Re, x_{k} \in \Re^{n}$, are respectively, the input, output, and state vector; $A_{k} \in \Re^{n \times n}, b_{k} \in \Re^{n}, c_{k} \in \Re^{1 \times n}$, and $d_{k} \in \Re$ are $N$-periodic, that is

$$
\begin{aligned}
A_{k+N} & =A_{k}, \quad b_{k+N}=b_{k}, \quad c_{k+N}=c_{k}, \quad \text { and } \\
d_{k+N} & =d_{k}, \quad k \geq 0 .
\end{aligned}
$$

We assume that filter (2.1) is (asymptotically) stable [1].

Consider the block diagram shown in Fig. 1. Let $r$ be the observed signal, which is the sum of the filter output $u$ and a measurement noise $v$, i.e.,

$$
r=u+v
$$

and let $\hat{s}$ be a $d$-step delay of the input $s$ to filter (2.1), that is

$$
\hat{s}_{k}= \begin{cases}s_{k-d}, & k \geq d \\ 0, & 0 \leq k<d .\end{cases}
$$

\footnotetext{
${ }^{1}$ Other models for periodic filters include polyphase representation, periodic difference equation, etc. The state equation seems most convenient for the problem addressed since there is an associated well-known formula for the MIMO time-invariant filter model.
}

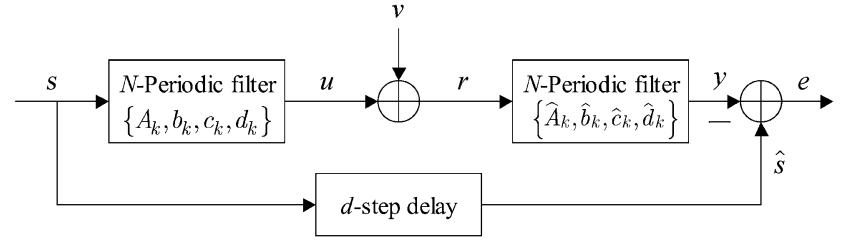

Fig. 1. Schematic description of periodic inverse filtering problem.

An approximate inverse of filter (2.1) is a causal stable $N$-periodic filter with input $r$ and output $y$ described by

$$
\begin{aligned}
w_{k+1} & =\hat{A}_{k} w_{k}+\hat{b}_{k} r_{k} \\
y_{k} & =\hat{c}_{k} w_{k}+\hat{d}_{k} r_{k}, \quad k \geq 0
\end{aligned}
$$

where $\hat{A}_{k}, \hat{b}_{k}, \hat{c}_{k}$, and $\hat{d}_{k}$ are $N$-periodic such that, with $x_{0}=$ $w_{0}=0$, the output $y$ is close to $\hat{s}$, i.e., the error signal

$$
e=\hat{s}-y
$$

is small for the input signal $s$ of interest.

The following assumptions are made in the sequel.

1) The input $s=\left\{s_{k} \in \Re, k \geq 0\right\}$ to filter (2.1) is a white sequence with zero-mean and unit variance.

2) The noise $v=\left\{v_{k} \in \Re, k \geq 0\right\}$ is a white sequence with zero-mean and variance $\sigma_{v}^{2}$ and is uncorrelated with the input $s$.

In this paper, we propose a method for constructing an approximate inverse of the form (2.5), with which the average energy of the block reconstruction error signal is kept small.

\section{B. Preliminary}

1) Transfer Matrix Representation of Periodic Filters: Consider again the filter (2.1). Define the block input $\bar{s}$, output $\bar{u}$, and state $\bar{x}$ as

$$
\begin{aligned}
& \bar{s}_{k}=\left[s_{k N} s_{k N+1} \ldots s_{k N+N-1}\right]^{T} \in \Re^{N} \\
& \bar{u}_{k}=\left[u_{k N} u_{k N+1} \ldots u_{k N+N-1}\right]^{T} \in \Re^{N}
\end{aligned}
$$

and

$$
\bar{x}_{k}=x_{k N} \text {. }
$$

There is an $N$-input $N$-output linear time-invariant system associated with filter (2.1) described by

$$
\begin{aligned}
\bar{x}_{k+1} & =\bar{A} \bar{x}_{k}+\bar{B} \bar{s}_{k} \\
\bar{u}_{k} & =\bar{C} \bar{x}_{k}+\bar{D} \bar{s}_{k}
\end{aligned}
$$

where $\bar{A} \in R^{n \times n}, \bar{B} \in R^{n \times N}, \bar{C} \in R^{N \times n}$, and $\bar{D} \in R^{N \times N}$, with $\bar{D}$ lower triangular, are such that, with $x_{0}=0$ and $\bar{x}_{0}=0$, the systems (2.1) and (2.8) have an identical I/O relation, except that in (2.8), the input and output are sequences of vectors of dimension $N$. The matrix $\bar{D}$ is lower triangular since filter (2.1) is causal [7, p. 1090]. Formulas for $\bar{A}, \bar{B}, \bar{C}$, and $\bar{D}$ can be found in [13]. The transfer matrix of the system (2.8) is

$$
\mathbf{G}(z)=\bar{C}(z I-\bar{A})^{-1} \bar{B}+\bar{D} .
$$

Thus, each linear causal $N$-periodic filter is represented by an $N \times N$ proper rational matrix with $\mathbf{G}(\infty)(=\bar{D})$ lower triangular. Conversely, any $N \times N$ proper rational matrix $\mathbf{G}(z)$ with 
$\mathbf{G}(\infty)$ lower triangular can be implemented as an $N$-periodic filter of the form (2.1) [7]. An algorithm for computing minimal $N$-periodic realizations can be found in [11]. In the sequel, we will simply call $\mathbf{G}(z)$ the transfer matrix of the periodic filter (2.1). We need the following results.

Proposition 2.1 [7]: If filter (2.1) is stable, then its transfer matrix $\mathbf{G}(z) \in \Re_{p}^{N \times N}(z)$. Conversely, each $\mathbf{G}(z) \in \Re_{p}^{N \times N}(z)$, with $\mathbf{G}(\infty)$ lower triangular, can be realized as an $N$-periodic filter of the form (2.1), which is stable.

Proposition 2.2 [10]: The transfer matrix of a $d$-step delay, viewed as an $N$-periodic system, is

$$
\mathbf{D}(z):=\left[\begin{array}{cc}
0 & z^{-1} I_{p} \\
I_{N-p} & 0
\end{array}\right] z^{-q} \in \Re_{p}^{N \times N}(z)
$$

where $d=p+q N, p$, and $q$ are non-negative integers with $0 \leq p \leq N-1$.

2) Some Properties of Real Rational Matrices in $\Re^{l \times m}(z)$ : Let $H$ be the space of all sequences of matrices $\mathrm{X}=\left\{\mathrm{X}_{k}\right\}, \mathrm{X}_{k} \in \Re^{l \times m}$, satisfying $\sum_{k=-\infty}^{\infty} \operatorname{Tr}\left[\mathrm{X}_{k}^{T} \mathrm{X}_{k}\right]=$ $\sum_{k=-\infty}^{\infty}\left\|\mathrm{X}_{k}\right\|_{F}^{2}<\infty$, where $\operatorname{Tr}[\cdot]$ and $\|\cdot\|_{F}$ denote, respectively, the trace and the Frobenius norm. Define the inner product on $H$ as

$$
\langle\mathrm{X}, \mathrm{Y}\rangle=\sum_{k=-\infty}^{\infty} \operatorname{Tr}\left[\mathrm{X}_{k}^{T} \mathrm{Y}_{k}\right], \quad \forall \mathrm{X}, \mathrm{Y} \in H
$$

which induces a norm $\|\cdot\|$ on $H:\|\mathrm{X}\|^{2}=\sum_{k=-\infty}^{\infty}\left\|\mathrm{X}_{k}\right\|_{F}^{2}$, for all $\mathrm{X} \in H$. Let $R H$ be the rational subspace of $H$, that is, if $\mathrm{X} \in R H$, then its $z$-transform

$$
\mathbf{X}(z)=\sum_{k=-\infty}^{\infty} \mathbf{X}_{k} z^{-k} \in \Re^{l \times m}(z)
$$

The region of convergence of $\mathbf{X}(z)$ in (2.12) is an annular region containing $\partial D$. If $\mathbf{X} \in R H$ is causal, i.e., $\mathbf{X}_{k}=0_{l \times m}$ for all $k<0$, then $\mathbf{X}(z)=\sum_{k=0}^{\infty} \mathbf{X}_{k} z^{-k} \in \Re_{p}^{l \times m}(z)$. The 2-norm of $\mathbf{X}(z) \in \Re^{l \times m}(z)$ is defined as

$$
\|\mathbf{X}\|_{2}:=\left(\frac{1}{2 \pi} \int_{-\pi}^{\pi} \operatorname{Tr}\left[\mathbf{X}\left(e^{j \theta}\right) \mathbf{X}^{*}\left(e^{j \theta}\right)\right] d \theta\right)^{1 / 2} .
$$

We say i) $\mathbf{X}(z) \in \Re^{l \times m}(z), l \geq m$, is inner if

$$
\mathbf{X}^{T}\left(z^{-1}\right) \mathbf{X}(z)=I_{m} .
$$

(ii) $\mathbf{Y}(z) \in \Re_{p}^{m \times m}(z)$ is outer if $\mathbf{Y}^{-1}(z) \in \Re_{p}^{m \times m}(z)$. Inner and outer rational matrices are sometimes called, respectively, allpass and minimum-phase. Note from (2.14) that if $\mathbf{X}(z) \in$ $\Re^{m \times m}(z)$ is square and inner, then $\mathbf{X}^{-1}(z)=\mathbf{X}^{T}\left(z^{-1}\right)$. For each $\mathbf{X}(z) \in \Re_{p}^{l \times m}(z)$ with $l \geq m$, there exists an inner $\mathbf{X}_{\mathbf{i}}(z) \in \Re_{p}^{l \times m}(z)$ and an outer $\mathbf{X}_{\mathbf{o}}(z) \in \Re_{p}^{m \times m}(z)$ such that [25]

$$
\mathbf{X}(z)=\mathbf{X}_{\mathbf{i}}(z) \mathbf{X}_{\mathbf{o}}(z) .
$$

The product expression (2.15) is called an inner-outer factorization of $\mathbf{X}(z)$ and is a generalization of the allpass-minimumphase factorization $[14$, p. 240] of scalar rational functions to rational matrix case. We need the following fact, which is the Parseval's relation for matrix sequences.

Proposition 2.3 [25]: Let $\mathrm{X} \in R H$ be the inverse $z$-transform of $\mathbf{X}(z) \in \Re^{l \times m}(z)$. Then, we have $\|\mathbf{X}\|_{2}=\|\mathbf{X}\|$.

\section{OPTIMALITY CRITERION}

In this section, we propose an optimality criterion for approximate inverse design. Our analysis is based on block signals and MIMO time-invariant models of periodic filters. As we will see, the characterization of the block reconstruction error is simple, and the selection of design criterion is natural and convenient.

Let the block reconstructed output $\bar{y}$, reconstruction error $\bar{e}$, and noise $\bar{v}$ be defined in an analog way as in (2.7a). Let $\mathbf{D}(z), \mathbf{G}(z)$, and $\mathbf{F}(z)$ be, respectively, the transfer matrices of the $d$-step delay [see (2.10)], filter (2.1), and filter (2.5); denote by $\mathrm{D}, \mathrm{G}$, and $\mathrm{F}$ the respective inverse $z$-transforms. Then, we have

$$
\bar{y}=\mathrm{F} * \mathrm{G} * \bar{s}+\mathrm{F} * \bar{v} .
$$

Since $\bar{e}=\mathrm{D} * \bar{s}-\bar{y}$ and from (3.1), we can write $\bar{e}$ as

$$
\bar{e}=\mathrm{D} * \bar{s}-\mathrm{F} * \mathrm{G} * \bar{s}-\mathrm{F} * \bar{v}=(\mathrm{D}-\mathrm{F} * \mathrm{G}) * \bar{s}-\mathrm{F} * \bar{v} .
$$

To reliably recover the input signal $\bar{s}$, we must keep the block reconstruction error $\bar{e}_{k}$ "small" for each $k \geq 0$. A commonly used measure of the "size" of $\bar{e}_{k}$ is the mean square value $E\left\|\bar{e}_{k}\right\|_{F}^{2}$, i.e., the average energy. The following proposition provides a characterization of this quantity. Specifically, we will see that, owing to the causality of the signal $s$ and noise $v, E\left\|\bar{e}_{k}\right\|_{F}^{2}$ is an increasing function of $k$ and asymptotically approaches a constant as $k \rightarrow \infty$. The result will allow a very natural optimal criterion for approximate inverse design. The proof of the proposition is given in Appendix A.

Proposition 3.1: Let the block error $\bar{e}$ be defined in the same way as in (2.7a). Then, we have

$$
E\left\|\bar{e}_{k}\right\|_{F}^{2}=\sum_{i=0}^{k}\left\|(\mathrm{D}-\mathrm{F} * \mathrm{G})_{i}\right\|_{F}^{2}+\sigma_{v}^{2} \sum_{j=0}^{k}\left\|\mathrm{~F}_{j}\right\|_{F}^{2} .
$$

In particular, $E\left\|\bar{e}_{k}\right\|_{F}^{2}$ increases with $k$ and, as $k \rightarrow \infty$, approaches

$$
J:=\|\mathbf{D}-\mathbf{F G}\|_{2}^{2}+\sigma_{v}^{2}\|\mathbf{F}\|_{2}^{2}
$$

Based on Proposition 3.1, we propose to construct an approximate inverse by minimizing the asymptotic block mean square error $J$ defined in (3.4). This is because if $J$ is small, then the monotone increasing property guarantees that $E\left\|\bar{e}_{k}\right\|_{F}^{2}$ is small for each $k \geq 0$. Moreover, the objective function will also allow a natural formulation of the optimization problem in terms of transfer matrices as an optimal model-matching problem, based on which a closed-form solution can be obtained. This will be shown in the next two sections.

\section{Remarks:}

a) We note that $\mathbf{F}(z) \mathbf{G}(z)$ is transfer matrix of the cascade connection of filters (2.1) and (2.5) [7, p. 1089]. As a result, the first term of the objective function $J$ in (3.4) can 
be regarded as a measure of the imperfectness of signal resolution (filter inversion). The second term represents the noise contribution to the approximation error with respect to a signal resolution quality attained by $\mathbf{F}(z)$.

b) We note from (3.4) that large noise variance $\sigma_{v}^{2}$ tends to emphasize large reduction in noise effect. However, this is done at the expense of the signal resolution quality. On the other hand, small $\sigma_{v}^{2}$ leads to better signal resolution quality but with smaller noise reduction. Hence, there is a tradeoff between signal resolution quality and noise reduction.

c) The problem of constructing an approximate inverse for a given periodic filter in the presence of noise is also addressed in [23] and [24]. By regarding the signal $s$ and noise $v$ as the input to the signal reconstruction system shown in Fig. 1 and error $e$ as the corresponding output, the optimality criterion adopted in [23] and [24] is to minimize the squared $\mathrm{H}_{2}$-norm of the I/O map from the augmented input $\left[\begin{array}{ll}s & v\end{array}\right]^{T}$ to the error $e$. In terms of block signals, such an objective function is shown to be equal to [23, p. 2698]

$$
\|[\mathbf{D}-\mathbf{F G} \quad \vdots \quad \mathbf{F}]\|_{2}^{2} .
$$

By the definition of the 2-norm [see (2.13)], it can be easily checked that the quantity given in (3.5) is a special case of the proposed objective function in (3.4) with noise variance fixed at $\sigma_{v}^{2}=1$. The resultant minimization problem in [23] and [24] is formulated in terms of the state equations associated with the augmented transfer matrix $[\mathbf{D}(z)-\mathbf{F}(z) \mathbf{G}(z) \vdots \mathbf{F}(z)]$ and is solved via the LMI-based approach.

\section{Optimal Model-Matching Problem}

\section{A. Problem Formulation}

From (3.4), it appears that an optimal $\mathbf{F}(z)$, if it exists, will tend to keep the two quantities $\|\mathbf{D}-\mathbf{F G}\|_{2}^{2}$ and $\|\mathbf{F}\|_{2}^{2}$ small or, equivalently, to keep $\mathbf{F}(z) \mathbf{G}(z) \approx \mathbf{D}(z)$ and $\mathbf{F}(z) \approx 0_{N \times N}$. The observation thus indicates that an optimal approximate inverse (which is stable and causal) can be obtained if we can find an $\mathbf{F}(z) \in \Re_{p}^{N \times N}(z)$, with $\mathbf{F}(\infty)$ lower triangular, such that the above two "matching" conditions hold as exact as possible. This suggests that we can formulate the problem as an optimal model-matching problem. Since such formulation involves only time-invariant transfer matrices, the related properties of rational matrices can be further used for deriving a solution.

To see this, we should note that the objective function $J$ in (3.4), which is a weighted sum of the two quantities $\|\mathbf{D}-\mathbf{F G}\|_{2}^{2}$ and $\|\mathbf{F}\|_{2}^{2}$, can be further rewritten by using only one single term. More precisely, by definition of 2-norm and from (3.4), we have

$$
J=\left\|\left[\mathbf{D}-\mathbf{F G} \quad \vdots \quad \sigma_{v} \mathbf{F}\right]\right\|_{2}^{2} .
$$

Define the following two augmented rational matrices:

$$
\hat{\mathbf{D}}(z):=\left[\begin{array}{lll}
\mathbf{D}(z) & \vdots & 0_{N \times N}
\end{array}\right] \in \Re_{p}^{N \times 2 N}(z)
$$

and

$$
\hat{\mathbf{G}}(z):=\left[\begin{array}{lll}
\mathbf{G}(z) & \vdots & -\sigma_{v} I_{N}
\end{array}\right] \in \Re_{p}^{N \times 2 N}(z) .
$$

From (4.1)-(4.3), we can express the objective function $J$ in the so-called model-matching form [2], [5], [6], as

$$
J=\|\hat{\mathbf{D}}-\mathbf{F} \hat{\mathbf{G}}\|_{2}^{2} .
$$

In (4.4), the rational matrix $\hat{\mathbf{D}}(z)$ represents the target "model" to be achieved: $\mathbf{D}(z)$ and $0_{N \times N}$ are the desired models, respectively, for signal resolution and noise reduction, whereas $\hat{\mathbf{G}}(z)$ is the given "plant" $\left(\mathbf{G}(z)\right.$ for signal, and $-\sigma_{v} I_{N}$ with respect to noise) based on which we are to design an $\mathbf{F}(z)$ to keep $\mathbf{F}(z) \hat{\mathbf{G}}(z)$ as close to $\hat{\mathbf{D}}(z)$ as possible in the 2-norm sense. The problem of optimal approximate inverse design, therefore, can be formulated as follows: Given a model $\hat{\mathbf{D}}(z)$ and a plant $\hat{\mathbf{G}}(z)$, find an $\mathbf{F}(z) \in \Re_{p}^{N \times N}(z)$, with $\mathbf{F}(\infty)$ lower triangular, such that the objective function $J$ in (4.4) is minimized.

Remarks:

a) Consider the noiseless case $\sigma_{v}^{2}=0$; hence, (4.4) reduces to

$$
J=\|\mathbf{D}-\mathbf{F G}\|_{2}^{2}
$$

We note that in this case, the model and plant $(\mathbf{D}(z)$ and $\mathbf{G}(z)$, respectively) are square rational matrices. If $\mathbf{G}^{-1}(z)$ has all its poles inside $\partial D$ and the reconstruction delay is large enough, it is shown in [10] that we can choose

$$
\mathbf{F}(z)=\mathbf{D}(z) \mathbf{G}^{-1}(z)
$$

to yield the reconstructed output $y$ identical to the $d$-step delay of the input $s$, viz., $y=\hat{s}$. We note that with $\mathbf{F}(z)$ in (4.6), the model $\mathbf{D}(z)$ is exactly matched in this case, and hence, $J=0$. The procedure for constructing one such $\mathbf{F}(z)$ with minimal possible reconstruction delay is given in [10, p. 198].

b) Formulation of other signal design and reconstruction problems as an optimal model-matching problem is also found in the designs of rate-changing multirate elements [2], [18], and perfect reconstruction filterbank, e.g., see [19] [where the same 2-norm criterion as in (4.4) is used], and [3], [5], [6] (in which an $H_{\infty}$ criterion is used). We should note, however, that the problems addressed in all these works do not consider the noise effect.

\section{B. Equivalent Problem with Lower Dimension}

Due to noise effect, the model and plant $(\hat{\mathbf{D}}(z)$ and $\hat{\mathbf{G}}(z)$, respectively) are nonsquare rational matrices. In what follows, based on an inner-outer factorization on $\hat{\mathbf{G}}^{T}(z)$, we will show that the proposed model-matching problem can be equivalently 
reduced to one in which both the model and plant are square rational matrices with lower dimensions. The reduction in dimension of model and plant will lead to a simple procedure for finding the optimal solution (in Section V). In addition, it will allow us to obtain a lower bound on the objective function. As we will see, this bound is an indication of the best achievable performance.

To be specific, write an inner-outer factorization of $\hat{\mathbf{G}}^{T}(z)$ as $\hat{\mathbf{G}}^{T}(z)=\mathbf{U}^{T}(z) \tilde{\mathbf{G}}^{T}(z)$, where $\mathbf{U}^{T}(z) \in \Re_{p}^{2 N \times N}(z)$ is inner and $\tilde{\mathbf{G}}^{T}(z) \in \Re_{p}^{N \times N}(z)$ is outer. Thus, we have

$$
\hat{\mathbf{G}}(z)=\tilde{\mathbf{G}}(z) \mathbf{U}(z) .
$$

Write

$$
\begin{aligned}
& \mathbf{U}(z)=\left[\mathbf{U}_{1}(z) \quad \vdots \quad \mathbf{U}_{2}(z)\right], \quad \text { where } \mathbf{U}_{j}(z) \in \Re_{p}^{N \times N}(z) \\
& 1 \leq j \leq 2 \text {. }
\end{aligned}
$$

Based on (4.4) and with (4.7) and (4.8), we have the following proposition, whose proof is given in Appendix B.

Proposition 4.1: Let $\mathbf{D}(z)$ defined in (2.10) be the transfer matrix associated with the $d$-step delay. Let the augmented transfer matrix $\hat{\mathbf{G}}(z)$ be defined in (4.3). Factorize $\hat{\mathbf{G}}(z)$ as in (4.7), and express $\mathbf{U}(z)$ as in (4.8). Then, the objective function $J$ can be expressed as

$$
J=\|\mathbf{H}-\mathbf{F} \tilde{\mathbf{G}}\|_{2}^{2}+\sigma_{v}^{2}\left\|\tilde{\mathbf{G}}^{-1}\right\|_{2}^{2}
$$

where

$$
\mathbf{H}(z):=\mathbf{D}(z) \mathbf{U}_{1}^{T}\left(z^{-1}\right) \in \Re^{N \times N}(z) .
$$

Based on (4.9), it follows immediately that, for a given $\mathbf{D}(z)$, the second term on the right-hand side (RHS) of (4.9) is independent of $\mathbf{F}(z)$ that is to be determined. Hence, to find an optimal $\mathbf{F}(z)$, it is equivalent to solving the following optimal model-matching problem, in which the model and plant are $N \times$ $N$ square rational matrices:

$$
\min \|\mathbf{H}-\mathbf{F} \tilde{\mathbf{G}}\|_{2}^{2} \text {, subject to } \mathbf{F}(z) \in \Re_{p}^{N \times N}(z) \text { and }
$$$$
\mathbf{F}(\infty) \text { is lower triangular. }
$$

\section{Remarks:}

a) We note that in the equivalent problem (4.11), the plant $\tilde{\mathbf{G}}(z)$ is an outer rational matrix. In the next section, this property will be further exploited for deriving the solution.

b) Consider the noiseless case, i.e., $\sigma_{v}^{2}=0$. Then, the factorization (4.7) is replaced by

$$
\mathbf{G}(z)=\mathbf{G}_{\mathbf{0}}(z) \mathbf{G}_{\mathbf{i}}(z)
$$

where $\mathbf{G}_{\mathbf{i}}^{T}(z) \mathbf{G}_{\mathbf{o}}^{T}(z)$ is an inner-outer factorization of $\mathbf{G}^{T}(z)$ (both $\mathbf{G}_{\mathbf{i}}(z)$ and $\mathbf{G}_{\mathbf{o}}(z)$ are square with dimension $N \times N)$. In this case, we have $\mathbf{H}(z)=\mathbf{D}(z) \mathbf{G}_{\mathbf{i}}^{T}\left(z^{-1}\right)$ instead, and (4.9) reduces to

$$
J=\left\|\mathbf{H}-\mathbf{F G}_{\mathbf{o}}\right\|_{2}^{2}
$$

c) Algorithms for computing an inner-outer factorization of $\hat{\mathbf{G}}^{T}(z)$ can be found in, e.g., [12], [19, p. 975], and [25, p. 555].

In addition, from (4.9), it can be easily seen that no matter which $\mathbf{F}(z)$ is used, a lower bound on the objective function $J$ is

$$
\sigma_{v}^{2}\left\|\tilde{\mathbf{G}}^{-1}\right\|_{2}^{2}
$$

Note that in noiseless case, the lower bound in (4.14) equals zero, and the objective function $J$ in (4.9) reduces to the one given in (4.13). Therefore, for a given noise variance $\sigma_{v}^{2}$, the bound in (4.14) can be regarded as the inherent approximation error incurred due to noise. In general, this lower bound can be asymptotically achieved as the value of reconstruction delay $d$ approaches infinity. To see this, we will first find an optimal solution by solving problem (4.11). This is done in the next section.

\section{OptIMAL SOLUTION}

In this section, we solve problem (4.11) for an optimal $\mathbf{F}(z)$. Section V-A reformulates problem (4.11) in the time-domain. Section V-B then derives a solution. Finally, Section V-C discusses some properties of the optimal solution.

\section{A. Optimization Problem in the Time-Domain}

To solve problem (4.11), we propose to rewrite the problem in time-domain and in terms of the inverse $z$-transforms of the related rational matrices. With such a time-domain formulation, there is a simple procedure for obtaining an optimal solution that satisfies the required constraints. To this end, we need some additional notations.

Recall that $R H$ is the space of all square summable sequences of real $l \times m$ matrices $\mathbf{X}=\left\{\mathrm{X}_{k}\right\}$ with $z$-transforms $\mathbf{X}(z) \in$ $\Re^{l \times m}(z)$. In the sequel, we set $l=m=N$. For integer $k_{0}$, let $R H_{k_{0}}^{+}:=\left\{\mathrm{X} \in R H \mid \mathrm{X}_{k}=0_{N \times N}, \forall k<k_{0}\right\}$, and $R H_{k_{0}}^{-}:=\left\{\mathrm{X} \in R H \mid \mathrm{X}_{k}=0_{N \times N}, \forall k \geq k_{0}\right\}$. Clearly, we have $R H=R H_{k_{0}}^{+} \stackrel{\perp}{\oplus} R H_{k_{0}}^{-}$. Note that $R H_{0}^{+}$is the subspace of causal sequences, and $R H_{0}^{-}$is the subspace of anti-causal sequences, respectively. For $\mathrm{X} \in R H$, let $\mathrm{P}_{k_{0}}^{+}[\mathrm{X}]$ be the projection of $\mathrm{X}$ onto $R H_{k_{0}}^{+}$and $\mathrm{P}_{k_{0}}^{-}[\mathrm{X}]$ be the projection of $\mathrm{X}$ onto $R H_{k_{0}}^{-}$. Thus, $\mathrm{X}=\mathrm{P}_{k_{0}}^{+}[\mathrm{X}]+\mathrm{P}_{k_{0}}^{-}[\mathrm{X}]$. Denote by $\delta$ the $N \times N$ matrix unit-impulse sequence, i.e., $\delta_{k}=I_{N}$ for $k=0$ and $\delta_{k}=0_{N \times N}$ otherwise.

Let $\mathrm{H}, \mathrm{F}$, and $\tilde{\mathrm{G}}$ be, respectively, the inverse $z$-transforms of $\mathbf{H}(z), \mathbf{F}(z)$, and $\tilde{\mathbf{G}}(z)$. Then, with Proposition 2.3, problem (4.11) is equivalent to

$$
\begin{aligned}
& \min \|\mathrm{H}-\mathrm{F} * \tilde{\mathrm{G}}\|^{2} \text {, subject to } \mathrm{F} \in R H_{0}^{+} \text {and } \\
& \qquad \mathrm{F}_{0}(=\mathbf{F}(\infty)) \text { is lower triangular. }
\end{aligned}
$$

\section{Remarks:}

a) We note that in problem (5.1), F and $\tilde{G}$ are causal sequences, but $\mathrm{H}$ is generally not. 
b) In the noiseless case, based on (4.12), the sequences $\mathrm{H}$ and $\tilde{G}$ in (5.1) are, respectively, the inverse $z$-transforms of $\mathbf{D}(z) \mathbf{G}_{\mathbf{i}}^{T}\left(z^{-1}\right)$ and $\mathbf{G}_{\mathbf{o}}(z)$.

\section{B. Optimal Solution}

The derivation of a solution is based on a rearrangement of the time-domain objective function $\|\mathrm{H}-\mathrm{F} * \tilde{\mathrm{G}}\|^{2}$. Specifically, it is shown in Appendix $\mathrm{C}$ that the quantity $\|\mathrm{H}-\mathrm{F} * \tilde{\mathrm{G}}\|^{2}$ can be decomposed as a sum of three terms as

$$
\begin{array}{r}
\|\mathrm{H}-\mathrm{F} * \tilde{\mathrm{G}}\|^{2}=\left\|\mathrm{P}_{0}^{-}[\mathrm{H}]\right\|^{2}+\left\|\mathrm{H}_{0}-\mathrm{F}_{0} \tilde{\mathrm{G}}_{0}\right\|_{F}^{2}+\| \mathrm{P}_{1}^{+}\left[\mathrm{H}-\mathrm{F}_{0} \tilde{\mathrm{G}}\right] \\
-\mathrm{P}_{1}^{+}[\mathrm{F}] * \tilde{\mathrm{G}} \|^{2} .
\end{array}
$$

We note that the first term on the RHS of (5.2), which is the energy of the anticausal component of $\mathrm{H}$, is independent of $\mathrm{F}$. In addition, since $\tilde{\mathbf{G}}(z)$ is outer, we have, by definition, $\tilde{\mathbf{G}}^{-1}(z) \in$ $\Re_{p}^{N \times N}(z)$ and, hence, its inverse $z$-transform $\tilde{\mathrm{G}}^{-1} \in R H_{0}^{+}$. As a result, for a given initial value $\mathrm{F}_{0}$, we can choose

$$
\mathrm{P}_{1}^{+}[\mathrm{F}]=\mathrm{P}_{1}^{+}\left[\mathrm{H}-\mathrm{F}_{0} \tilde{\mathrm{G}}\right] * \tilde{\mathrm{G}}^{-1} \in R H_{1}^{+}
$$

to make the third term on the RHS of (5.2) equal to zero. Hence, if we can first find the lower triangular matrix $\bar{F}_{0}$, the initial value, which minimizes the second term on the RHS of (5.2), namely

$$
\left\|\mathrm{H}_{0}-\mathrm{F}_{0} \tilde{\mathrm{G}}_{0}\right\|_{F}^{2}
$$

then the quantity $\|\mathrm{H}-\mathrm{F} * \tilde{\mathrm{G}}\|^{2}$ is minimized by choosing $\mathrm{P}_{1}^{+}[\mathrm{F}]$ as in (5.3) and $F_{0}=\bar{F}_{0}$.

The optimal $\overline{\mathrm{F}}_{0}$ is constructed as follows. Factorize $\tilde{\mathrm{G}}_{0}^{T}$ as

$$
\tilde{\mathrm{G}}_{0}^{T}=Q L^{T},
$$

where $Q$ is unitary, and $L$ is lower triangular. This is simply a QR-factorization of the matrix $\tilde{\mathrm{G}}_{0}^{T}$. Since $\tilde{\mathbf{G}}^{-1}(z) \in \Re_{p}^{N \times N}(z), \quad \tilde{\mathbf{G}}_{0}(=\tilde{\mathbf{G}}(\infty))$ is nonsingular, as is $L$. Write $\mathrm{H}_{0} Q=\left[t_{i j}\right]$, where $t_{i j}$ is the $i j$ th entry $1 \leq i, j \leq N$. Define the lower triangular matrix $W=\left[w_{i j}\right]$ by

$$
w_{i j}= \begin{cases}t_{i j}, & i \geq j \\ 0, & i<j .\end{cases}
$$

We note that the matrix $W$ thus defined is simply obtained from $\mathrm{H}_{0} Q$ by nullimg its entries on the upper diagonals. Then, the optimal $\overline{\mathrm{F}}_{0}$ is given in the following proposition.

Proposition 5.1: The lower triangular matrix that minimizes the Frobenius norm (5.4) is $\overline{\mathrm{F}}_{0}=W L^{-1}$, where $W$ and $L$ are, respectively, defined in (5.6) and (5.5).

Proof: Since, from (5.5), $\tilde{\mathrm{G}}_{0}=L Q^{T}=L Q^{-1}$ and the Frobenius norm is unitary invariant, we have

$$
\begin{aligned}
\left\|\mathrm{H}_{0}-\mathrm{F}_{0} \tilde{\mathrm{G}}_{0}\right\|_{F}^{2} & =\left\|\mathrm{H}_{0}-\mathrm{F}_{0} L Q^{-1}\right\|_{F}^{2}=\left\|\left(\mathrm{H}_{0} Q-\mathrm{F}_{0} L\right) Q^{-1}\right\|_{F}^{2} \\
& =\left\|\mathrm{H}_{0} Q-\mathrm{F}_{0} L\right\|_{F}^{2} .
\end{aligned}
$$

Since $\mathrm{F}_{0} L$ is lower triangular, it follows from (5.7) that the minimum norm is attained if we choose $F_{0} L$ to eliminate the entries on the main and lower diagonals of $\mathrm{H}_{0} Q$. This immediately implies that $\overline{\mathrm{F}}_{0} L=W$, and the result follows.

With (5.3) and $\bar{F}_{0}$ in Proposition 5.1, the optimal $\bar{F}$ is thus $\overline{\mathrm{F}}=\overline{\mathrm{F}}_{0} \delta+\mathrm{P}_{1}^{+}\left[\mathrm{H}-\overline{\mathrm{F}}_{0} \tilde{\mathrm{G}}\right] * \tilde{\mathrm{G}}^{-1}$. With some rearrangement, the expression of $\bar{F}$ can be further simplified as

$$
\overline{\mathrm{F}}=\left\{\overline{\mathrm{F}}_{0} \tilde{\mathrm{G}}_{0} \delta+\mathrm{P}_{1}^{+}[\mathrm{H}]\right\} * \tilde{\mathrm{G}}^{-1} .
$$

By taking the $z$-transform of both sides of (5.8), the transfer matrix of the optimal approximate inverse is immediately obtained as

$$
\overline{\mathbf{F}}(z)=\left\{\overline{\mathbf{F}}_{0} \tilde{\mathbf{G}}(\infty)+\mathbf{H}_{1}^{+}(z)\right\} \tilde{\mathbf{G}}^{-1}(z)
$$

where $\mathbf{H}_{1}^{+}(z)$ is the $z$-transform of $P_{1}^{+}[\mathrm{H}]$.

Remark: The proposed optimal approximate inverse $\overline{\mathbf{F}}(z)$ in (5.9) is, in general, IIR. One way to obtain a $j$ th-order FIR approximate inverse is to keep the first $j+1$ terms of $\bar{F}$ to get the FIR transfer matrix

$$
\overline{\mathbf{F}}_{j}(z)=\sum_{n=0}^{j} \overline{\mathbf{F}}_{n} z^{-n}
$$

\section{Some Properties of the Optimal Approximate Inverse}

With optimal $\overline{\mathbf{F}}(z)$ in (5.9), it can be verified, using (5.2) and Proposition 2.3, that

$$
\|\mathbf{H}-\overline{\mathbf{F}} \tilde{\mathbf{G}}\|_{2}^{2}=\left\|\mathbf{H}_{0}^{-}\right\|_{2}^{2}+\left\|\mathrm{H}_{0} Q-W\right\|_{F}^{2}
$$

where $\mathbf{H}_{0}^{-}(z)$ is the $z$-transform of the anticausal sequence $P_{0}^{-}[\mathrm{H}]$. From (4.9) and (5.11), the minimal value of the objective function $J$ is thus

$$
J_{\min }=\left\|\mathbf{H}_{0}^{-}\right\|_{2}^{2}+\left\|\mathrm{H}_{0} Q-W\right\|_{F}^{2}+\sigma_{v}^{2}\left\|\tilde{\mathbf{G}}^{-1}\right\|_{2}^{2} .
$$

Note that since $\mathbf{U}_{1}(z) \in \Re_{p}^{N \times N}(z), \mathbf{U}_{1}^{T}\left(z^{-1}\right)$ has all its poles outside $\partial D$, and therefore, its inverse $z$-transform is an infinite-duration sequence belonging to $R H_{1}^{-}$. Since $\mathbf{D}(z)$ defined in (2.10) is the transfer matrix of the $d$-step delay, the sequence $\mathrm{H}$, which is the inverse $z$-transform of $\mathbf{H}(z)=\mathbf{D}(z) \mathbf{U}_{1}^{T}\left(z^{-1}\right)$, is thus a delayed version of the one associated with $\mathbf{U}_{1}^{T}\left(z^{-1}\right)$. Hence, as the reconstruction delay $d$ is sufficiently large, we can make the sequence $P_{1}^{-}[\mathrm{H}]$ arbitrarily close to a zero sequence and, hence, the two quantities $\left\|\mathbf{H}_{0}^{-}\right\|_{2}^{2}$ and $\left\|\mathrm{H}_{0} Q-W\right\|_{F}^{2}$ arbitrarily close to zero. From (5.12), we thus have the following asymptotic property.

Proposition 5.2: The minimal value of the objective function $J_{\min } \rightarrow \sigma_{v}^{2}\left\|\tilde{\mathbf{G}}^{-1}\right\|_{2}^{2}$ as the reconstruction delay $d \rightarrow \infty$. For the special case $\sigma_{v}^{2}=0$, the limit value of $J_{\min }$ is zero.

From Proposition 5.2, the quantity $\sigma_{v}^{2}\left\|\tilde{\mathbf{G}}^{-1}\right\|_{2}^{2}$ can therefore be regarded as the "best achievable" performance for a given noise variance $\sigma_{v}^{2}$. In particular, if, for some choice of delay, the resultant $J_{\min }$ is substantially larger than this quantity, we can further improve the performance by introducing more delays. Since increasing the delay will, at the same time, increase the order of $\mathbf{H}_{1}^{+}(z)$, and hence the order of optimal $\overline{\mathbf{F}}(z)$ [see (5.9)], there is a tradeoff between the achievable performance 
and the order of the transfer matrix associated with the optimal approximate inverse.

Remark: Consider the noiseless case $\sigma_{v}^{2}=0$. Assume that $\mathbf{G}(z)$ has a nonminimum phase zero $z_{0}$ (viz., $z_{0}$ lying outside $\partial D$ ). From (4.12) and since $\mathbf{G}_{\mathbf{o}}(z)$ is outer, $z_{0}$ must be a zero of the inner factor $\mathbf{G}_{\mathbf{i}}(z)$ and, hence, a pole of $\mathbf{G}_{\mathbf{i}}^{-1}(z)$. Since $\mathbf{G}_{\mathbf{i}}(z)$ is square inner when there is no noise, we have $\mathbf{G}_{\mathbf{i}}^{T}\left(z^{-1}\right)=\mathbf{G}_{\mathbf{i}}^{-1}(z)$ [by definition (2.14)]. This immediately implies that $\mathbf{H}(z)=\mathbf{D}(z) \mathbf{G}_{\mathbf{i}}^{T}\left(z^{-1}\right)=\mathbf{D}(z) \mathbf{G}_{\mathbf{i}}^{-1}(z)$; the sequence $H$ is thus a delayed version of $G_{i}^{-1}$ (the inverse $z$-transform of $\mathbf{G}_{\mathbf{i}}^{-1}(z)$ ). Since $z_{0}$ is a pole of $\mathbf{G}_{\mathbf{i}}^{-1}(z), \mathbf{G}_{\mathbf{i}}^{-1}$ has an anticausal component of infinite duration. If $z_{0}$ is close to $\partial D$, then the significant part of the anticausal component of $\mathrm{G}_{\mathbf{i}}^{-1}$ will last a long time. As a result, it would require a large reconstruction delay and, therefore, a high order $\overline{\mathbf{F}}(z)$ to keep $\mathrm{P}_{1}^{-}[\mathrm{H}]$ close to a zero sequence and, hence, achieve a good approximation performance. For the case $\sigma_{v}^{2}>0$, although a nonminimum phase zero $z_{0}$ of $\mathbf{G}(z)$ is no longer a pole of $\mathbf{U}_{1}^{T}\left(z^{-1}\right)$, simulation results (see Simulation 4) show that this tendency, however, seems to hold.

\section{EXTENSION To MIMO $N$-Periodic Systems}

Suppose that the state equations (2.1) and (2.5), instead, represent two $N$-periodic systems, each with $K$ inputs and $K$ outputs. Our goal is to design an approximate inverse (2.5) of system (2.1) such that, for $1 \leq l \leq K$, the signal in the $l$ th output channel of system (2.5) is close to a $d_{l}$-step delay of the $l$ th input signal of system (2.1). Assume that i) the $K$ input signals to system (2.1) are uncorrelated white sequences and that ii) the $K$ additive noises are uncorrelated white sequences and are uncorrelated with the input signals to system (2.1). Under these assumptions, the proposed method for the scalar case can be extended. This is seen as follows.

Note that each $K$-input $K$-output $N$-periodic system of the form (2.1) is represented by a rational matrix $\mathbf{G}(z) \in \Re_{p}^{K N \times K N}(z)$ with $\mathbf{G}(\infty) K \times K$-block lower triangular (and vice versa) [7]. The optimality criterion in terms of transfer matrices described in Section III thus applies; the objective function is of the same form as in (3.4), except that the transfer matrices involved are of dimensions $K N \times K N$. By following the same procedures as in Section IV, it amounts to solving a model-matching problem as in (4.11), subject to $\mathbf{F}(z) \in \Re_{p}^{K N \times K N}(z)$ and $\mathbf{F}(\infty)$ being $K \times K$-block lower triangular. Note that the transfer matrix $\mathbf{D}(z)$, in this case, is associated with the $K$-input $K$-output time-invariant system whose $l$ th output is a $d_{l}$-step delay of the $l$ th input $1 \leq l \leq K$ (one way to construct such a $\mathbf{D}(z)$ is given in Appendix $\mathrm{D}$ ). Since the problem is essentially the same with the one in the SISO case, except for the constraint on $\mathrm{F}_{0}(=\mathbf{F}(\infty))$, we still need to determine the $K \times K$-block lower triangular matrix $\overline{\mathrm{F}}_{0}$ that minimizes the Frobenius norm (5.4). To this end, factorize $\tilde{\mathrm{G}}_{0}^{T}$ as in (5.5). Since $L$ is lower triangular, $\mathrm{F}_{0} L$ is $K \times K$-block lower triangular. Equation (5.7) shows that the optimal $\overline{\mathrm{F}}_{0}$ is chosen so that $\overline{\mathrm{F}}_{0} L$ eliminates the entries on the $K \times K$-block main and lower diagonals of the matrix $\mathrm{H}_{0} Q$. This implies that $\overline{\mathrm{F}}_{0}=W L^{-1}$, where $W \in \Re^{K N \times K N}$ is the $K \times K$-block lower triangular matrix obtained from $\mathrm{H}_{0} Q$ by nulling its entries on the $K \times K$-block upper diagonals [ $W$ is constructed similarly as in (5.6)].

\section{SimUlation RESUlTS}

In this section, we use several numerical simulations to illustrate the performance of the proposed optimal approximate inverse. In our simulations, we estimate the block mean squared error at time $k$, viz., $E\left\|\bar{e}_{k}\right\|_{F}^{2}$, via the time-average

$$
\varepsilon_{k}:=\frac{1}{I} \sum_{i=1}^{I}\left\|\bar{e}_{k}^{i}\right\|_{F}^{2}
$$

where $I$ is the total number of independent Monte Carlo realizations, and $\bar{e}_{k}^{i}$ is the $k$ th block error sample in the $i$ th realization. Based on the computed $\varepsilon_{k}$, we determine the associated steady-state region by selecting a time instant $k_{0}$ such that $\varepsilon_{k}$ approaches a constant for all $k \geq k_{0}$. The estimated value of the asymptotic block mean squared error $J$ in (3.4) is then computed as

$$
\hat{J}:=\frac{1}{S} \sum_{k=k_{0}}^{k_{0}+S} \varepsilon_{k}
$$

where $S$ is the total number of estimated block mean squared errors in the steady state. For each conducted Monte Carlo realization, the input to filter (2.1) is a white Gaussian sequence with 100 samples. The signal-to-noise ratio (SNR) is defined as SNR: $=10 \log _{10}\left(\sigma_{v}^{-2}\right)$. In all simulations, the number of trials is $I=1000$. To compute the optimal approximate inverse, we use the algorithm in $[25$, p. 555] to compute an inner-outer factorization of the rational matrix $\hat{\mathbf{G}}^{T}(z)$.

1) Simulation 1-Comparision with Previous Work [10]: The performance of the proposed optimal approximate inverse is compared with that obtained by the minimal-delay inverse filter constructed in the noiseless case [10]. We consider the 2-periodic filter described as

$$
\begin{aligned}
A_{0} & =\left[\begin{array}{cc}
0 & 0.5 \\
-0.5 & 0
\end{array}\right], \quad A_{1}=\left[\begin{array}{ll}
1 & 1 \\
1 & 2
\end{array}\right] ; \quad b_{0}=\left[\begin{array}{c}
0 \\
-0.5
\end{array}\right] \\
b_{1} & =\left[\begin{array}{l}
1 \\
0
\end{array}\right] ; \quad c_{0}=\left[\begin{array}{l}
1 \\
0
\end{array}\right]^{T}, \quad c_{1}=\left[\begin{array}{l}
1 \\
1
\end{array}\right]^{T} \\
d_{0} & =1, \quad d_{1}=-0.5 .
\end{aligned}
$$

The associated transfer matrix is

$\mathbf{G}(z)=\frac{1}{z^{2}+0.25}\left[\begin{array}{cc}z(z-0.5) & z-0.5 \\ -0.5 z(z+0.5) & -0.5\left(z^{2}+z+0.75\right)\end{array}\right]$.

By computations, $\mathbf{G}^{-1}(z)$ is proper and has all its poles inside $\partial D$. According to [10], the minimal achievable reconstruction delay is zero, and the transfer matrix associated with the inverse filter is simply $\mathbf{G}^{-1}(z)$ [cf. (4.6)]. For a fair comparison, the proposed optimal approximate inverse based on (5.9) is implemented by setting $d=0\left(\mathbf{D}(z)=I_{2}\right)$. Fig. 2 shows the respective computed objective function $\hat{J}$ with respect to different values of SNR. As we can see from the figure, for SNR $>25$ $\mathrm{dB}$, the performances obtained by the two methods are roughly 


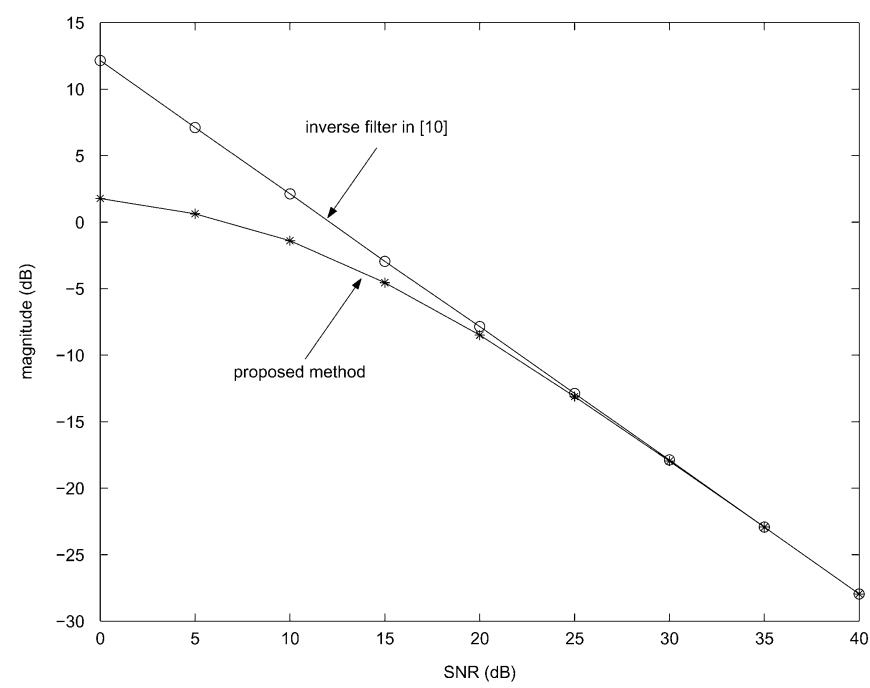

Fig. 2. Computed $\hat{J}$ versus SNR: Proposed method versus method in [10].

the same. For SNR $<25 \mathrm{~dB}$, the proposed method leads to improved performance since it does the task of noise reduction. For the particular case SNR $=15 \mathrm{~dB}$, the transfer matrix of the optimal approximate inverse is computed as in (7.5), shown at the bottom of the page. Using the algorithm in [11], a minimal 2-periodic realization of $\overline{\mathbf{F}}(z)$ in (7.5) is obtained as

$$
\begin{aligned}
& \hat{A}_{0}=\left[\begin{array}{cc}
0.4151 & 0.7849 \\
0 & 0.0703
\end{array}\right], \quad \hat{A}_{1}=\left[\begin{array}{cc}
-0.143 & -0.8227 \\
0.591 & -0.5169
\end{array}\right] \\
& \hat{b}_{0}=\left[\begin{array}{c}
-1.1471 \\
0.5336
\end{array}\right], \quad \hat{b}_{1}=\left[\begin{array}{c}
0.8569 \\
-0.1879
\end{array}\right] \\
& \hat{c}_{0}=\left[\begin{array}{c}
1.2265 \\
-1.3805
\end{array}\right]^{T}, \quad \hat{c}_{1}=\left[\begin{array}{c}
0.7939 \\
0.2367
\end{array}\right]^{T} \\
& \hat{d}_{0}=0.7994, \quad \hat{d}_{1}=-1.69 .
\end{aligned}
$$

2) Simulation 2-Comparison with the LMI-Based Method [23], [24]: The performance of the proposed approximate inverse is compared with that obtained by the LMI approach in [23] and [24]. We consider the following 2-periodic FIR filter, which is used in simulation in [23] and [24]:

$$
\begin{aligned}
A_{0}=A_{1} & =\left[\begin{array}{lll}
0 & 1 & 0 \\
0 & 0 & 1 \\
0 & 0 & 0
\end{array}\right] ; \quad b_{0}=b_{1}=\left[\begin{array}{l}
0 \\
0 \\
1
\end{array}\right] \\
c_{0} & =\left[\begin{array}{c}
-1 \\
2 \\
1
\end{array}\right]^{T}, \quad c_{1}=\left[\begin{array}{c}
1 \\
-2 \\
2
\end{array}\right]^{T} ; \quad d_{0}=5, \quad d_{1}=3
\end{aligned}
$$

and use both methods to design an FIR approximate inverse. At each SNR level, an optimal approximate inverse is first designed according to (5.9) (we choose reconstruction delay $d=0$ as in

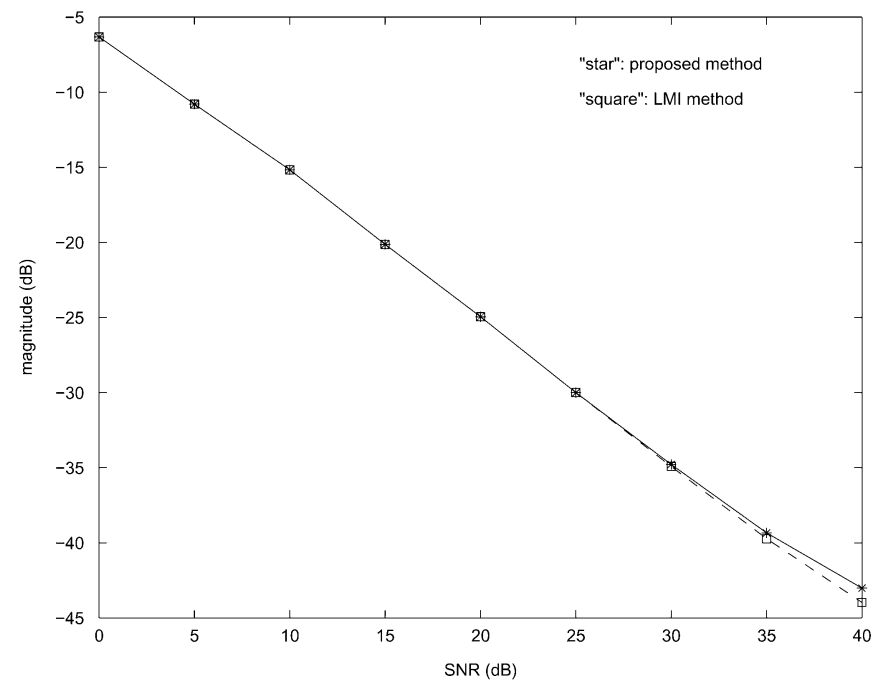

Fig. 3. Computed $\hat{J}$ versus SNR: FIR approximate inverse filters obtained by two methods.

the simulations in [23] and [24]). Then, a ninth-order FIR approximate inverse $\overline{\mathbf{F}}_{9}(z)$ is obtained using (5.10) (computations show that this choice yields a good match in performance to the computed optimal IIR $\overline{\mathbf{F}}(z)$ at each SNR). By computations, the dimension of a minimal realization of the obtained $\overline{\mathbf{F}}_{9}(z)$, at each SNR, is 19. For a fair comparison, an FIR 2-periodic filter with the same dimension of realization is designed based on [24]. In particular, we obtain the LMI-based solution by properly scaling a parameter in the design equation (the $\tilde{D}_{k}$ matrix in $[24$, p. 1688, (3.11)]) by noise standard deviation to further reflect the actual noise variance; the solution thus obtained then minimizes the same objective function as ours. Fig. 3 shows the respective computed $\hat{J}$ versus SNR. From the figure, we can see that the performances of the proposed analytical solution and the one based on the LMI method are roughly the same; this is not unexpected since both solutions tend to minimize the same objective function.

3) Simulation 3-Effects of Reconstruction Delay: In this simulation, we demonstrate the effect of reconstruction delay. In particular, we will show that when the optimal $\overline{\mathbf{F}}(z)$ is used, the computed objective function $\hat{J}$, as the delay $d$ increases, will be close to the lower bound (4.14). We consider the 2-periodic filter obtained via replacing the $c_{0}$ vector in state (7.3) by $c_{0}=\left[\begin{array}{ll}13 & 3\end{array}\right.$. We fix the SNR at $20 \mathrm{~dB}$. For each $0 \leq d \leq 9$, the optimal $\overline{\mathbf{F}}(z)$ is implemented. Fig. 4 shows the respective computed $\hat{J}$ versus delay $d$. In addition, at each delay, the associated theoretical minimal value $J_{\min }$ based on (5.12) and lower bound (4.14) are calculated and shown in the figure. The result shows that the experimental values $\hat{J}$ are very close to the theoretical $J_{\min }$ and exhibit the expected tendency.

4) Simulation 4-Effect of Nonminimum Phase Zero of $\mathbf{G}(z)$ : In this simulation, we demonstrate the dependence of

$$
\overline{\mathbf{F}}(z)=\frac{1}{z^{2}-0.3683 z+0.01634}\left[\begin{array}{cc}
0.7994 z^{2}+0.6851 z+0.5274 & 1.31 z-0.6851 \\
-\left(0.7844 z^{2}+0.4119 z\right) & -\left(1.69 z^{2}-0.7844 z+0.01046\right)
\end{array}\right] .
$$




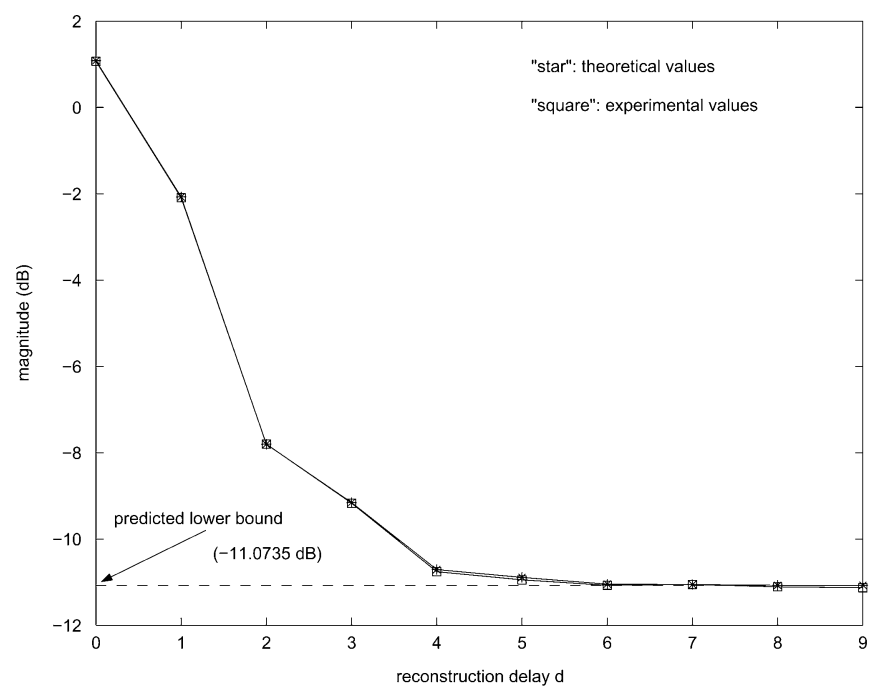

Fig. 4. Computed $\hat{J}$ and theoretical values versus delay. $\mathrm{SNR}=20 \mathrm{~dB}$.

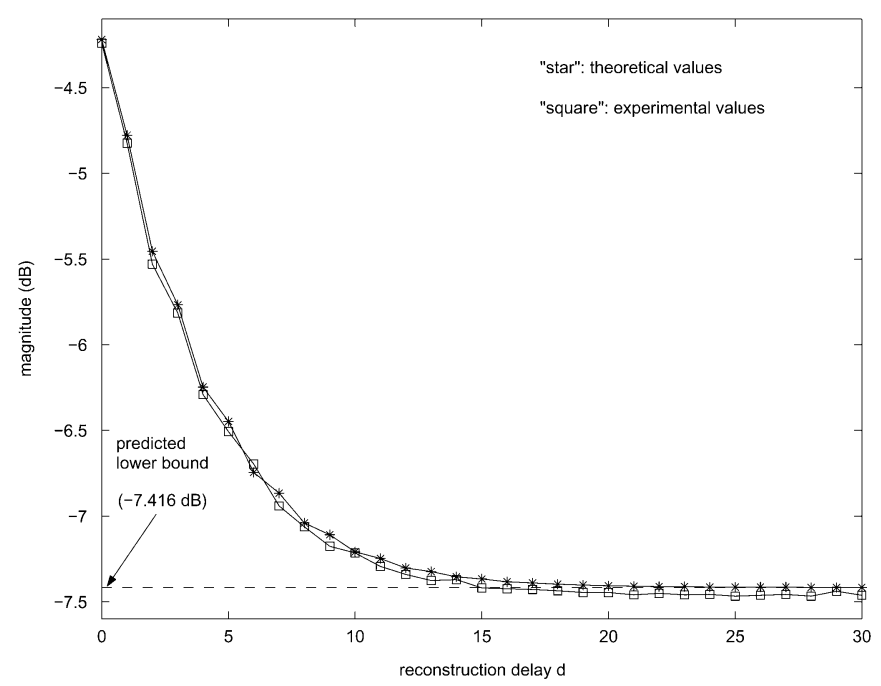

Fig. 5. Computed $\hat{J}$ and theoretical values versus delay, filter with zero at $Z=-1.08, \mathrm{SNR}=20 \mathrm{~dB}$.

performance on the locations of nonminimum phase zeros of $\mathbf{G}(z)$. We consider the filter used in the previous simulation and another filter obtained by replacing the $c_{0}$ vector in state (7.3) by $c_{0}=\left[\begin{array}{ll}1 & 0.58\end{array}\right]$ (in the sequel, we will call them "filter (a)" and "filter (b)," respectively). By computations, the respective transfer matrices have a single nonminimum phase zero at $z_{0}=3.5$ and $z_{0}=1.08$. For fixed SNR at $20 \mathrm{~dB}$, Fig. 5 shows the computed $\hat{J}$ for filter (b), together with the corresponding theoretical values $J_{\min }$ and the lower bound, at various values of delay $d$. From Figs. 4 and 5 , we can see that for filter (b), the value of delay required to keep $\hat{J}$ close to the lower bound is relatively large. If fact, based on the theoretical values $J_{\min }$ for each filter, the required delay such that $\left|J_{\min }-\sigma_{v}^{2}\left\|\tilde{\mathbf{G}}^{-1}\right\|_{2}^{2}\right| \leq 0.0005$ are, respectively, $d=6$ for filter (a) and $d=28$ for filter (b); the orders of respective optimal $\overline{\mathbf{F}}(z)$ are 3 and 14 . Hence, if $\mathbf{G}(z)$ contains a nonminimum phase zero close to $\partial D$, a large delay and, hence, an approximate inverse of high order, would be required

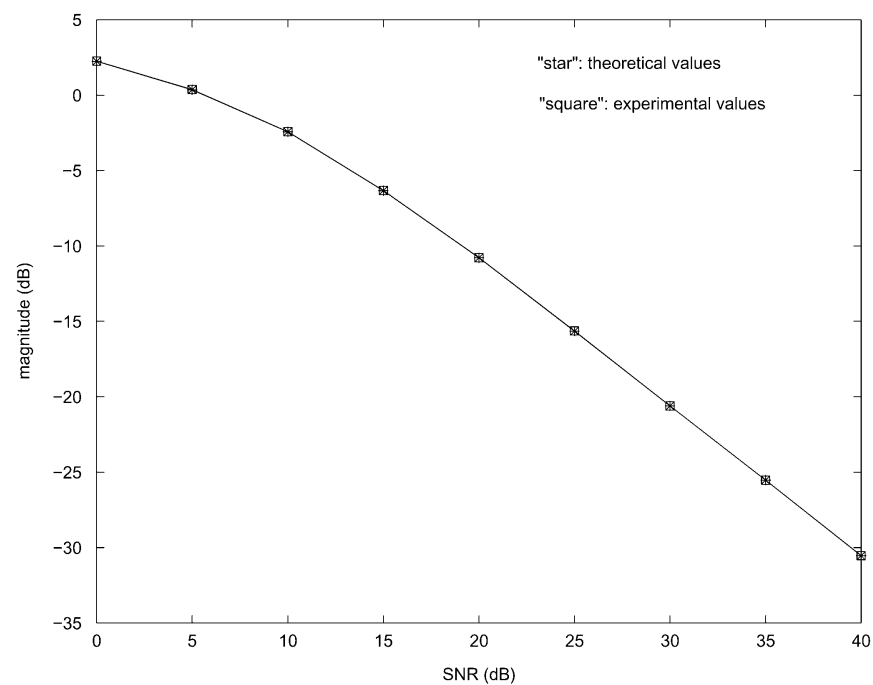

Fig. 6. Computed $\hat{J}$ and theoretical values versus SNR (MIMO case).

to achieve an approximation error close to the lower bound $\sigma_{v}^{2}\left\|\tilde{\mathbf{G}}^{-1}\right\|_{2}^{2}$.

5) Simulation 5-MIMO Periodic Systems: In this simulation, we demonstrate the performance of the proposed method when it applies to MIMO periodic systems. We consider the 2-input 2-output 2-periodic system given as

$$
\begin{aligned}
A_{0} & =\left[\begin{array}{cc}
0 & 0.5 \\
-0.5 & 0
\end{array}\right], \quad A_{1}=\left[\begin{array}{ll}
1 & 1 \\
1 & 2
\end{array}\right] ; \quad b_{0}=\left[\begin{array}{cc}
1 & -6 \\
0 & 0
\end{array}\right] \\
b_{1} & =\left[\begin{array}{ll}
1 & 0 \\
0 & 1
\end{array}\right] ; \quad c_{0}=\left[\begin{array}{ll}
1 & 2 \\
1 & 0
\end{array}\right], \quad c_{1}=\left[\begin{array}{cc}
1 & 1 \\
0 & 1
\end{array}\right] \\
d_{0} & =\left[\begin{array}{ll}
1 & 0 \\
2 & 1
\end{array}\right], \quad d_{1}=\left[\begin{array}{ll}
1 & 1 \\
0 & 0
\end{array}\right] .
\end{aligned}
$$

An approximate inverse of system (7.8) is designed based on (5.9); the reconstruction delays in channels 1 and 2 are, respectively, 6 and 7. The input signal to each channel is a white sequence of 100 samples. The resultant vector error signal (of dimension 2) is obtained, and the (experimental) objective function $\hat{J}$ is computed. Fig. 6 shows the computed $\hat{J}$ and the theoretical $J_{\min }$ versus SNR. The result shows that the experimental values are very close to the theoretical values.

\section{CONCLUSION}

We study the problem of constructing an approximate inverse of SISO linear causal discrete-time periodic filters in the presence of measurement noise. In terms of block signals and block time-invariant filter model, the characterization of the block error signal is simple, and the selection of design criterion is very natural and convenient. The proposed optimality criterion, which minimizes the asymptotic block mean square error, allows us to formulate the problem in terms of transfer matrices as an optimal model-matching problem. The problem is solved using an inner-outer factorization and in terms of related time-domain sequences. In particular, the proposed time-domain approach for a solution yields a simple procedure for obtaining a closed-form optimal transfer matrix subject to the causality constraint. The computations 
required are inner-outer factorizations for rational matrices and QR-factorizations but do not involve numerical optimization (as is required in existing LMI method). We also obtain a lower bound incurred by noise on the objective function, which can be considered as the "target performance" for a given noise variance. The bound can be asymptotically achieved as the order of optimal transfer matrix increases. In fact, there is a tradeoff between the achievable performance and the order of the solution. The optimal transfer matrix is, in general, IIR, but an FIR approximate inverse can simply be obtained by truncating the associated inverse $z$-transform. The proposed method is also extended to MIMO periodic systems.

APPENDIX A

PROOF OF PROPOSITION 3.1

From (3.2) and since both $s$ and $v$ are causal, we have

$$
\bar{e}_{k}=\sum_{i=0}^{k}(\mathrm{D}-\mathrm{F} * \mathrm{G})_{i} \bar{s}_{k-i}-\sum_{j=0}^{k} \mathrm{~F}_{j} \bar{v}_{k-j}, \quad \forall k \geq 0 .
$$

Note that

$$
\begin{aligned}
E\left\|\bar{e}_{k}\right\|_{F}^{2} & =E \bar{e}_{k}^{T} \bar{e}_{k}=E \operatorname{Tr}\left[\bar{e}_{k}^{T} \bar{e}_{k}\right]=E \operatorname{Tr}\left[\bar{e}_{k} \bar{e}_{k}^{T}\right] \\
& =\operatorname{Tr}\left[E \bar{e}_{k} \bar{e}_{k}^{T}\right] .
\end{aligned}
$$

By substituting $\bar{e}_{k}$ in (A.1) into (A.2) and since $\bar{s}$ and $\bar{v}$ are mutually uncorrelated white vector processes, (3.3) follows. With (3.3), we immediately have, for any $k \geq 0$

$$
\begin{aligned}
E\left\|\bar{e}_{k+1}\right\|_{F}^{2} & -E\left\|\bar{e}_{k}\right\|_{F}^{2} \\
& =\left\|(\mathrm{D}-\mathrm{F} * \mathrm{G})_{k+1}\right\|_{F}^{2}+\sigma_{v}^{2}\left\|\mathrm{~F}_{k+1}\right\|_{F}^{2} \geq 0
\end{aligned}
$$

and hence, $E\left\|\bar{e}_{k}\right\|_{F}^{2}$ is an increasing function in $k$. In addition, letting $k \rightarrow \infty$ in (3.3), we have $E\left\|\bar{e}_{\infty}\right\|_{F}^{2}=\|\mathrm{D}-\mathrm{F} * \mathrm{G}\|^{2}+$ $\sigma_{v}^{2}\|\mathrm{~F}\|^{2}$. The result (3.4) then follows by Proposition 2.3.

\section{APPENDIX B}

\section{PROOF OF PROPOSITION 4.1}

To prove Proposition 4.1, we need the following two lemmas.

Lemma B.1 ([22, p. 213]: Let $\mathbf{U}^{T}(z) \in \Re_{p}^{2 N \times N}(z)$ be the inner rational matrix defined in (4.7). Then, there exists an inner rational matrix $\mathbf{U}_{\perp}^{T}(z) \in \Re_{p}^{2 N \times N}(z)$, called the complementary inner factor of $\mathbf{U}^{\bar{T}}(z)$, such that

$$
\mathbf{V}^{T}(z):=\left[\mathbf{U}^{T}(z) \quad \vdots \quad \mathbf{U}_{\perp}^{T}(z)\right] \in \Re_{p}^{2 N \times 2 N}(z)
$$

is square and inner. In particular, we have

$$
\mathbf{U}_{\perp}(z) \mathbf{U}_{\perp}^{T}\left(z^{-1}\right)=I_{N} \quad \text { and } \quad \mathbf{U}(z) \mathbf{U}_{\perp}^{T}\left(z^{-1}\right)=0_{N \times N} .
$$

Lemma B.2 [25]: Let $\mathbf{X}(z) \in \Re^{l \times l}(z)$ and $\mathbf{Y}(z) \in$ $\Re^{m \times m}(z)$ be two square inner rational matrices. Then, for any $\mathbf{W}(z) \in \Re^{l \times m}(z)$, we have $\|\mathbf{X W}\|_{2}=\|\mathbf{W Y}\|_{2}=\|\mathbf{W}\|_{2}$, that is, pre or post multiplying a rational matrix by a square inner factor preserves 2-norm.
Write

$$
\begin{aligned}
\mathbf{U}_{\perp}^{T}(z) & =\left[\begin{array}{lll}
\mathbf{U}_{\perp 1}(z) & \vdots & \mathbf{U}_{\perp 2}(z)
\end{array}\right]^{T}, \quad \text { where } \mathbf{U}_{\perp j}(z) \\
& \in \Re_{p}^{N \times N}(z) .
\end{aligned}
$$

We first claim

$$
J=\|\mathbf{H}-\mathbf{F} \tilde{\mathbf{G}}\|_{2}^{2}+\|\mathbf{E}\|_{2}^{2}, \quad \text { where } \mathbf{E}(z):=\mathbf{U}_{\perp 1}^{T}\left(z^{-1}\right) .
$$

It can be shown that

$$
\|\mathbf{E}\|_{2}^{2}=\sigma_{v}^{2}\left\|\tilde{\mathbf{G}}^{-1}\right\|_{2}^{2} .
$$

The result thus follows from (B.4) and (B.5).

Proof of Equation (B.4): From (4.4) and (4.7), we have $J=\|\hat{\mathbf{D}}-\mathbf{F} \tilde{\mathbf{G}} \mathbf{U}\|_{2}^{2}$. Write

$$
\begin{aligned}
& \hat{\mathbf{D}}(z)-\mathbf{F}(z) \tilde{\mathbf{G}}(z) \mathbf{U}(z) \\
& =\hat{\mathbf{D}}(z)-\left[\mathbf{F}(z) \tilde{\mathbf{G}}(z) \quad \vdots 0_{N \times N}\right] \underbrace{\left[\begin{array}{c}
\mathbf{U}(z) \\
\cdots \\
\mathbf{U}_{\perp}(z)
\end{array}\right]}_{=\mathbf{V}(z) \text { in }(\mathrm{B} .1)} .
\end{aligned}
$$

Since $\mathbf{V}(z)$ in (B.1) is square and inner, from (B.6), it follows that

$$
\begin{aligned}
& \hat{\mathbf{D}}(z)-\mathbf{F}(z) \tilde{\mathbf{G}}(z) \mathbf{U}(z)
\end{aligned}
$$

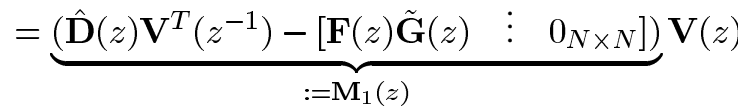

$$
\begin{aligned}
& =\mathbf{M}_{1}(z) \mathbf{V}(z) .
\end{aligned}
$$

From (B.7) and by Lemma B.2, we immediately have $J=$ $\left\|\mathbf{M}_{1}\right\|_{2}^{2}$, and hence, we must still find an expression of $\left\|\mathbf{M}_{1}\right\|_{2}^{2}$. Since $\mathbf{V}^{T}\left(z^{-1}\right)=\left[\mathbf{U}^{T}\left(z^{-1}\right) \mathbf{U}_{\perp}^{T}\left(z^{-1}\right)\right.$ ] [this follows from (B.1)], we can express the rational matrix $\mathbf{M}_{1}(z)$ defined in (B.7) as

$$
\mathbf{M}_{1}(z)=\left[\hat{\mathbf{D}}(z) \mathbf{U}^{T}\left(z^{-1}\right)-\mathbf{F}(z) \tilde{\mathbf{G}}(z) \quad \vdots \quad \hat{\mathbf{D}}(z) \mathbf{U}_{\perp}^{T}\left(z^{-1}\right)\right] .
$$

By definition of $\hat{\mathbf{D}}(z)$ in (4.2), and with $\mathbf{U}(z)$ and $\mathbf{U}_{\perp}(z)$ given as in (4.7) and (B.3), we have $\hat{\mathbf{D}}(z) \mathbf{U}^{T}\left(z^{-1}\right)=\mathbf{D}(z) \mathbf{U}_{1}^{T}\left(z^{-1}\right)=\mathbf{H}(z)$ and $\hat{\mathbf{D}}(z) \mathbf{U}_{\perp}^{T}\left(z^{-1}\right)=\mathbf{D}(z) \mathbf{U}_{\perp 1}^{T}\left(z^{-1}\right)$. Hence, $\mathbf{M}_{1}(z)$ in (B.8) becomes

$$
\mathbf{M}_{1}(z)=\left[\mathbf{H}(z)-\mathbf{F}(z) \tilde{\mathbf{G}}(z) \quad \vdots \quad \mathbf{D}(z) \mathbf{U}_{\perp 1}^{T}\left(z^{-1}\right)\right] .
$$

With (B.9) and by the definition of 2-norm [see (2.13)], we thus have

$$
J=\|\mathbf{H}-\mathbf{F} \tilde{\mathbf{G}}\|_{2}^{2}+\|\mathbf{D E}\|_{2}^{2}=\|\mathbf{H}-\mathbf{F} \tilde{\mathbf{G}}\|_{2}^{2}+\|\mathbf{E}\|_{2}^{2}
$$

where the last equality follows since $\mathbf{D}(z)$ in (2.10), which is the transfer matrix of the $d$-step delay, is square and inner and by using Lemma B.2. 

write

Proof of Equation (B.5): From (4.3) and (4.7), we can

$$
\mathbf{U}(z)=\left[\tilde{\mathbf{G}}^{-1}(z) \mathbf{G}(z) \quad \vdots \quad-\sigma_{v} \tilde{\mathbf{G}}^{-1}(z)\right] .
$$

With $\mathbf{U}(z)$ and $\mathbf{U}_{\perp}(z)$ given as in (B.11) and (B.3) and since $\mathbf{U}(z) \mathbf{U}_{\perp}^{T}\left(z^{-1}\right)=0_{N \times N}$ [see (B2)], it can be easily checked that $\mathbf{U}_{\perp 2}^{T}\left(z^{-1}\right)=\sigma_{v}^{-1} \mathbf{G}(z) \mathbf{U}_{\perp 1}^{T}\left(z^{-1}\right)$, and hence, again with (B.3), we have

$$
\mathbf{U}_{\perp}^{T}\left(z^{-1}\right)=\left[\begin{array}{c}
I_{N} \\
\cdots \\
\sigma_{v}^{-1} \mathbf{G}(z)
\end{array}\right] \mathbf{U}_{\perp 1}^{T}\left(z^{-1}\right) .
$$

From (B.12) and since $\mathbf{U}_{\perp}(z) \mathbf{U}_{\perp}^{T}\left(z^{-1}\right)=I_{N}$ [see (B.2)], we have

$$
\sigma_{v}^{-1} \mathbf{U}_{\perp 1}(z) \underbrace{\left\{\sigma_{v}^{2} I_{N}+\mathbf{G}^{T}\left(z^{-1}\right) \mathbf{G}(z)\right\}}_{:=\mathbf{Q}(z)} \sigma_{v}^{-1} \mathbf{U}_{\perp_{1}}^{T}\left(z^{-1}\right)=I_{N} .
$$

Let $\mathbf{S}(z) \in \Re_{p}^{N \times N}(z)$ be the spectral factor [22, p. 213] of $\mathbf{Q}(z)$, that is, $\mathbf{Q}(z)=\mathbf{S}^{T}\left(z^{-1}\right) \mathbf{S}(z), \forall z$. From (B.13), it follows that

$$
\tilde{\mathbf{U}}(z):=\sigma_{v}^{-1} \mathbf{S}(z) \mathbf{U}_{\perp 1}^{T}\left(z^{-1}\right) \in \Re^{N \times N}(z)
$$

is square and inner. Since, from (B.14), $\mathbf{E}(z)=\mathbf{U}_{\perp 1}^{T}\left(z^{-1}\right)=$ $\sigma_{v} \mathbf{S}^{-1}(z) \tilde{\mathbf{U}}(z)$ and $\tilde{\mathbf{U}}(z)$ is square and inner, we have (from Lemma B.2) that

$$
\|\mathbf{E}\|_{2}^{2}=\sigma_{v}^{2}\left\|\mathbf{S}^{-1}\right\|_{2}^{2}=\frac{\sigma_{v}^{2}}{2 \pi} \int_{-\pi}^{\pi} \operatorname{Tr}\left[\mathbf{S}^{-1}\left(e^{j \theta}\right) \mathbf{S}^{-*}\left(e^{j \theta}\right)\right] d \theta .
$$

Since $\mathbf{S}(z)$ is a spectral factor of $\mathbf{Q}(z)$ (i.e., $\mathbf{Q}(z)=$ $\left.\mathbf{S}^{T}\left(z^{-1}\right) \mathbf{S}(z), \forall z\right)$, we have, for any $\theta \in[02 \pi]$,

$$
\begin{aligned}
\mathbf{S}^{-1}\left(e^{j \theta}\right) \mathbf{S}^{-*}\left(e^{j \theta}\right) & =\left[\mathbf{S}^{*}\left(e^{j \theta}\right) \mathbf{S}\left(e^{j \theta}\right)\right]^{-1}=\mathbf{Q}^{-1}\left(e^{j \theta}\right) \\
& =\left(\sigma_{v}^{2} I_{N}+\mathbf{G}^{*}\left(e^{j \theta}\right) \mathbf{G}\left(e^{j \theta}\right)\right)^{-1}
\end{aligned}
$$

where the last equality follows by definition of $\mathbf{Q}(z)$ [see (B.13)]. With (B.15) and (B.16), we immediately have

$$
\|\mathbf{E}\|_{2}^{2}=\frac{\sigma_{v}^{2}}{2 \pi} \int_{-\pi}^{\pi} \operatorname{Tr}\left[\left(\sigma_{v}^{2} I_{N}+\mathbf{G}^{*}\left(e^{j \theta}\right) \mathbf{G}\left(e^{j \theta}\right)\right)^{-1}\right] d \theta .
$$

Since, for any $\theta \in\left[\begin{array}{ll}0 & 2 \pi\end{array}\right]$, the two (positive definite) matrices $\sigma_{v}^{2} I_{N}+\mathbf{G}^{*}\left(e^{j \theta}\right) \mathbf{G}\left(e^{j \theta}\right)$ and $\sigma_{v}^{2} I_{N}+\mathbf{G}\left(e^{j \theta}\right) \mathbf{G}^{*}\left(e^{j \theta}\right)$ have the same eigenvalues, so do the respective inverse matrices. This implies $\operatorname{Tr}\left\{\left(\sigma_{v}^{2} I_{N}+\mathbf{G}^{*}\left(e^{j \theta}\right) \mathbf{G}\left(e^{j \theta}\right)\right)^{-1}\right\}=$ $\operatorname{Tr}\left\{\left(\sigma_{v}^{2} I_{N}+\mathbf{G}\left(e^{j \theta}\right) \mathbf{G}^{*}\left(e^{j \theta}\right)\right)^{-1}\right\}$, and hence, from (B.17), we have

$$
\|\mathbf{E}\|_{2}^{2}=\frac{\sigma_{v}^{2}}{2 \pi} \int_{-\pi}^{\pi} \operatorname{Tr}\left[\left(\sigma_{v}^{2} I_{N}+\mathbf{G}\left(e^{j \theta}\right) \mathbf{G}^{*}\left(e^{j \theta}\right)\right)^{-1}\right] d \theta .
$$

From (4.3) and (4.7), and since $\mathbf{U}^{T}(z)$ is inner, we have, for all $\theta \in\left[\begin{array}{ll}0 & 2 \pi\end{array}\right]$

$\hat{\mathbf{G}}\left(e^{j \theta}\right) \hat{\mathbf{G}}^{*}\left(e^{j \theta}\right)=\sigma_{v}^{2} I_{N}+\mathbf{G}\left(e^{j \theta}\right) \mathbf{G}^{*}\left(e^{j \theta}\right)=\tilde{\mathbf{G}}\left(e^{j \theta}\right) \tilde{\mathbf{G}}^{*}\left(e^{j \theta}\right)$.

The result follows from (B.18) and (B.19).

APPENDIX C

DERIVATION OF (5.2)

Since $\mathrm{F} * \tilde{\mathrm{G}}=\sum_{n=0}^{\infty} \mathrm{F}_{n} \mathrm{z}^{-n} \tilde{\mathrm{G}}$, we have

$$
\begin{aligned}
\mathrm{H}-\mathrm{F} * \tilde{\mathrm{G}} & =\left(\mathrm{H}-\mathrm{F}_{0} \tilde{\mathrm{G}}\right)-\sum_{n=1}^{\infty} \mathrm{F}_{n} \mathrm{z}^{-n} \tilde{\mathrm{G}} \\
& =\left(\mathrm{H}-\mathrm{F}_{0} \tilde{\mathrm{G}}\right)-\mathrm{P}_{1}^{+}[\mathrm{F}] * \tilde{\mathrm{G}} .
\end{aligned}
$$

Decompose the first term on the RHS of (C.1) as $\mathrm{H}-\mathrm{F}_{0} \tilde{\mathrm{G}}=$ $\mathrm{P}_{1}^{-}\left[\mathrm{H}-\mathrm{F}_{0} \tilde{\mathrm{G}}\right]+\mathrm{P}_{1}^{+}\left[\mathrm{H}-\mathrm{F}_{0} \tilde{\mathrm{G}}\right]$, and hence, with (C.1), we have

$$
\mathrm{H}-\mathrm{F} * \tilde{\mathrm{G}}=\mathrm{P}_{1}^{-}\left[\mathrm{H}-\mathrm{F}_{0} \tilde{\mathrm{G}}\right]+\underbrace{\left(\mathrm{P}_{1}^{+}\left[\mathrm{H}-\mathrm{F}_{0} \tilde{\mathrm{G}}\right]-\mathrm{P}_{1}^{+}[\mathrm{F}] * \tilde{\mathrm{G}}\right)}_{\in R H_{1}^{+}} \text {. }
$$

Write

$$
\begin{aligned}
\mathrm{P}_{1}^{-}\left[\mathrm{H}-\mathrm{F}_{0} \tilde{\mathrm{G}}\right] & =\mathrm{P}_{0}^{-}\left[\mathrm{H}-\mathrm{F}_{0} \tilde{\mathrm{G}}\right]+\left(\mathrm{H}_{0}-\mathrm{F}_{0} \tilde{\mathrm{G}}_{0}\right) \delta \\
& =\mathrm{P}_{0}^{-}[\mathrm{H}]+\left(\mathrm{H}_{0}-\mathrm{F}_{0} \tilde{\mathrm{G}}_{0}\right) \delta
\end{aligned}
$$

where the last equality follows since $\mathrm{P}_{0}^{-}\left[\mathrm{H}-\mathrm{F}_{0} \tilde{\mathrm{G}}\right]=\mathrm{P}_{0}^{-}[\mathrm{H}]$ (for $\mathrm{F}_{0} \tilde{\mathrm{G}}$ is causal). Substituting $\mathrm{P}_{1}^{-}\left[\mathrm{H}-\mathrm{F}_{0} \tilde{\mathrm{G}}\right]$ in (C.3) into (C.2) and by definition of norm $\|\cdot\|$ in (2.11), the result follows.

\section{APPENDIX D}

\section{TRANSFER MATRIX OF MIMO DELAY}

For a fixed $1 \leq m \leq K$, we will show how to construct the transfer matrix $\mathbf{D}_{m}(\bar{z}) \in \Re_{p}^{K N \times K N}(z)$ associated with the $K$-input $K$-output system whose $\mathrm{I} / \mathrm{O}$ relation is a $d_{m}$-step delay in the $m$ th channel. Since a MIMO delay can be considered as a cascade of $K$ systems, each of which being a delay in a single channel, the associated transfer matrix is then obtained as $\prod_{l=1}^{K} \mathbf{D}_{l}(z)$ [7].

We first note that $\mathbf{D}_{m}(z)=I_{K N}$ if $d_{m}=0$. If $d_{m} \neq 0$, write $d_{m}=p_{m}+q_{m} N$, where $0 \leq p_{m} \leq N-1$. The resultant $\mathbf{D}_{m}(z)$ can be obtained from $I_{K N}$ by replacing its $(i K+m)$ th rows $0 \leq i \leq N-1$, respectively, by

$$
\left[\begin{array}{cc}
0 & z^{-1} I_{p_{m}} \\
I_{N-p_{m}} & 0
\end{array}\right] z^{-q_{m}}\left[\begin{array}{c}
\mathrm{e}_{m}^{T} \\
\vdots \\
\mathrm{e}_{(N-1) K+m}^{T}
\end{array}\right]
$$

where $\mathrm{e}_{i K+m} \in \Re^{K N}$ is the $(i K+m)$ th standard unit vector $0 \leq i \leq N-1$. The result (D.1) can be verified by investigating the $z$-transforms of the block input and output [defined similarly as in (2.7a) but is of dimension $K N$ ] associated with the system and using (2.10). 


\section{REFERENCES}

[1] F. M. Callier and C. A. Desoer, Linear System Theory. New York: Springer-Verlag, 1991.

[2] W. M. Campbell and T. W. Parks, "Design of a class of multirate systems using a maximum relative $l^{2}$-error criterion," IEEE Trans. Signal Processing, vol. 45, pp. 561-571, Mar. 1997.

[3] T. Chen and B. A. Francis, "Design of multirate filter banks by $\mathrm{H}_{\infty}$ optimization," IEEE Trans. Signal Processing, vol. 43, pp. 2822-2830, Dec. 1995.

[4] G. Gelli and F. Verde, "Two-stage interference-resistant adaptive periodically time-varying CMA blind equalization," IEEE Trans. Signal Processing, vol. 50, pp. 662-672, Mar. 2002.

[5] J. Huang and G. Gu, "A direct approach to the design of QMF banks via frequency domain optimization," IEEE Trans. Signal Processing, vol. 46, pp. 2131-2138, Aug. 1998.

[6] J. Huang, G. Gu, and B. A. Shenoi, "Design of multichannel QMF banks via frequency domain optimization," IEEE Trans. Circuits Syst. II, vol. 46, pp. 599-607, May 1999.

[7] P. P. Khargonekar, K. Poolla, and A. Tannenbaum, "Robust control of linear time-invariant plants using periodic compensation," IEEE Trans. Automatic Control, vol. AC-30, pp. 1088-1096, Nov. 1985.

[8] K. Kazlauskas, "Inversions of periodically time-varying digital filters," IEEE Trans. Circuits Syst. II, vol. 41, pp. 173-175, Feb. 1994.

[9] C. W. King and C. A. Lin, "A unified approach to scrambling filter design," IEEE Trans. Signal Processing, vol. 43, pp. 1753-1765, Aug. 1995.

[10] C. A. Lin and C. W. King, "Inverting periodic filters," IEEE Trans. Signal Processing, vol. 42, pp. 196-200, Jan. 1994.

[11] - "Minimal periodic realization of transfer matrices," IEEE Trans. Automat. Contr., vol. 38, pp. 462-466, Mar. 1993.

[12] Z. Lin, B. M. Chen, and Y. Shamash, "Inner-outer factorization of discrete-time transfer function matrices," IEEE Trans. Circuits Syst. I, vol. 43, pp. $941-945$, Nov. 1996.

[13] R. A. Meyer and C. S. Burrus, "A unified analysis of multirate and periodically time-varying digital filters," IEEE Trans. Circuits and Systems, vol. CAS-22, pp. 162-168, 1975.

[14] A. V. Oppenheim and R. W. Schafer, Discrete-Time Signal Processing. Englewood Cliffs, NJ: Prentice-Hall, 1989.

[15] A. G. Orozco-Lugo and D. C. Mclernon, "An application of linear periodically time-varying digital filters to blind equalization," in Proc. IEE Colloq. Digital Filters: Enabling Technol., London, U.K., Apr. 1998, pp. 11/1-11/6.

[16] J. S. Prater and C. M. Loeffler, "Analysis and design of periodically time-varying IIR filters, with applications to transmultiplexing," IEEE Trans. Signal Processing, vol. 40, pp. 2715-2725, Nov. 1992.

[17] E. Serpedin and G. B. Giannakis, "Blind channel identification and equalization with modulation-induced cyclostationarity," IEEE Trans. Signal Processing, vol. 47, pp. 1930-1944, July 1999.

[18] R. G. Shenoy, D. Burnside, and T. W. Parks, "Linear periodic systems and multirate filter design," IEEE Trans. Signal Processing, vol. 42, pp. 2242-2256, Sept. 1994.
[19] M. S. Spurbeck and C. T. Mullis, "Least squares approximation of perfect reconstruction filter banks," IEEE Trans. Signal Processing, vol. 46, pp. 968-978, Apr. 1998

[20] P. P. Vaidyanathan, Multirate Systems and Filter Banks. Englewood Cliffs, NJ: Prentice-Hall, 1993.

[21] M. Vetterli, "Invertibility of periodically time-varying filters," IEEE Trans. Circuits Syst., vol. 36, pp. 148-150, Jan. 1989.

[22] M. Vidyasagar, Control System Synthesis: A Factorization Approach. Cambridge, MA: MIT Press, 1985.

[23] S. Wang, L. Xie, and C. Zhang, " $\mathrm{H}_{2}$ optimal inverse of periodic FIR digital filters," IEEE Trans. Signal Processing, vol. 48, pp. 2696-2700, Sept. 2000

[24] H. Zhou, L. Xie, and C. Zhang, "A direct approach to $\mathrm{H}_{2}$ optimal deconvolution of periodic digital channels," IEEE Trans. Signal Processing, vol. 50, pp. 1685-1698, July 2002.

[25] K. Zhou, J. C. Doyle, and K. Glover, Robust and Optimal Control. Englewood Cliffs, NJ: Prentice-Hall, 1996.

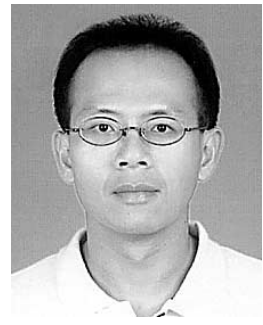

Jwo-Yuh Wu was born in Tainan, Taiwan, R.O.C. in 1973. He received the B.S. degree in 1996, the M.S. degree in 1998, and the Ph.D. degree in 2002 from the National Chiao Tung University, Hsinchu, Taiwan, all in electrical and control engineering.

$\mathrm{He}$ is currently a post doctor researcher with the Department of Communication Engineering, National Chiao Tung University. His current research interests are in signal processing.

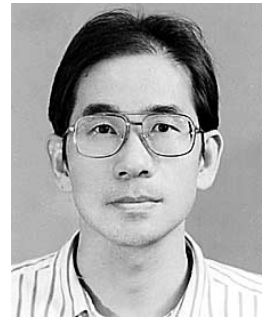

Ching-An Lin received the B.S. degree from the National Chiao Tung University, Hsinchu, Taiwan, R.O.C., in 1977, the M.S. degree from the University of New Mexico, Albuquerque, in 1980, and the $\mathrm{Ph} . \mathrm{D}$. degree from the University of California, Berkeley, in 1984, all in electrical engineering.

He was with the Chung Shan Institute of Science and Technology, Taoyan, Taiwan, from 1977 to 1979 and with Integrated Systems Inc., Palo Alto, CA, from 1984 to 1986. Since June 1986, he has been with the Department of Electrical and Control Engineering, the National Chiao Tung University, where he is a Professor. His current research interests are in control and signal processing. 